\title{
Behaviour of metals at ultra-high strain rate by using femtosecond laser shockwaves
}

\author{
J.-P. Cuq-Lelandais ${ }^{1}$, M. Boustie ${ }^{2}$, L. Berthe ${ }^{3}$, and T. De Rességuier ${ }^{2}$ \\ ${ }^{1}$ CEA-DAM, Centre de Valduc, 21120 Is-sur-Tille, France \\ 2 Institut PPRIME, UPR 3346 CNRS-ENSMA-Université de Poitiers, 86961 Futuroscope Cedex, France \\ ${ }^{3}$ Arts et Métiers ParisTech, 150 Bd. de l'Hôpital, 75013 Paris, France
}

\begin{abstract}
The mechanical behavior of materials under extreme conditions can be investigated by using laser driven shocks. Actually, femtosecond (fs) technologies allow to reach strong pressures over a very fast duration. This work is dedicated to characterize metals behavior in this ultra-short mode, (aluminum, tantalum), leading to an extreme dynamic solicitation in the target $\left(>10^{7} \mathrm{~s}^{-1}\right)$. The study includes the validation of experimental results obtained on the LULI 100TW facility by comparison with numerical model. Three modeling steps are considered. First, we characterize the pressure loading resulting from the fs lasermatter interaction, different from what happens in the classical nanosecond regime. Then, the shock wave propagation is observed through the target and particularly its pressure decay, strong in this regime. The elastic-plastic influence on the shock attenuation is discussed, particularly for tantalum which has a high elastic limit. Dynamic damage appears with spallation. Experimentally, spallation is characterized by VISAR measurements and post-test observations. Shots with different thicknesses have been carried out to determine the damage properties in function of strain rate. We show in this work that a simple instantaneous rupture criterion is not sufficient to reproduce the damage induced in the sample. Only the Kanel model, which includes damage kinetics, is able to reproduce experimental data (VISAR measurements, spall thickness). A generalization of this model to any strain rate can be performed by confronting these results to other shock generators data (ns laser driven shocks, plate impacts). One remarkable result is that every Kanel parameters follows a power law with strain rate in dynamic regime $\left(10^{5}\right.$ to $\left.10^{8} \mathrm{~s}^{-1}\right)$ for both aluminum and tantalum.
\end{abstract}

\section{Introduction}

Dynamic behaviour and damage investigation in materials present many interests for research applications and some industry fields (aeronautic, automobile, defense...). These aspects can be studied by generating high pressure shock waves [1]. One method in particular consists in using high energy laser short pulses to generate a pressure wave $[2,3]$. The characteristic durations involved are mainly about the nanosecond range and strain rates about $10^{7} \mathrm{~s}^{-1}$. The main advantage of such process is the possibility of recovering the shocked sample to analyze the spalled damaged zones, contrary to more conventional methods as plate impacts or explosions. The latest laser technologies evolutions provide an access to shorter regimes in durations, going below the picosecond [4]. This kind of regime is leading to extreme dynamic solicitations in the target, and spallation at a micrometric scale [5].

In this work, samples shocked by femtosecond (fs) laser are investigated. The main goal is to characterize dynamic damage in these extreme conditions and calibrate a rupture model in this regime. The study is divided into three steps: first, the pressure loading induced by the laser interaction with the solid medium is numerically predicted. Then, the shock wave propagation will be discussed and finally the damage model (Kanel) will be dimensioned in order to fit experimental data (velocity measurements, post-mortem observations). In addition, the parameters in femtosecond regime are compared to the values obtained with other spall generators (plate impact, nanosecond laser) to see the evolution with strain rate.

\section{Experimental setup}

In this work, spallation by laser driven shock experiments have been performed on the femtosecond LULI' ${ }^{\text {' }}$ s "100TW" facility in 2010 (now called ELFIE), with a laser beam of $1.06 \mu \mathrm{m}$ wavelength, up to 30J energy and a Gaussian pulse of $300 \mathrm{fs}$ full width at half maximum (See Figure 1). The laser is focused on metallic samples tantalum and aluminum - of various thicknesses (20 to $500 \mu \mathrm{m}$ ), with a $2 \mathrm{~mm}$ impact diameter. In this configuration, we can obtain laser intensities up to $3 \mathrm{PW} / \mathrm{cm}^{2}$ $\left(1 \mathrm{PW}=10^{15} \mathrm{~W}\right)$. As a time resolved diagnosis, a VISAR measurement of free surface velocity is used [3]. The data obtained can inform about the shock propagation and damage history by comparing it with numerical results computed by numerical simulations. In this case, a good numerical free surface velocity restitution is the key to validate one dynamic damage model and determine its parameters.

\section{Generation \& propagation of fs laser driven shocks}

\subsection{Pressure loading generated by Laser/Matter interaction}

Laser shockwaves generation is ruled by the laser-matter interaction. Previous studies have shown the generation

\footnotetext{
${ }^{\text {a }}$ LULI = Laboratoire d'Utilisation des Laser Intenses - UMR CNRS 7605 (Ecole Polytechnique, France)

Website : http//www.luli-polytechnique.fr
} 


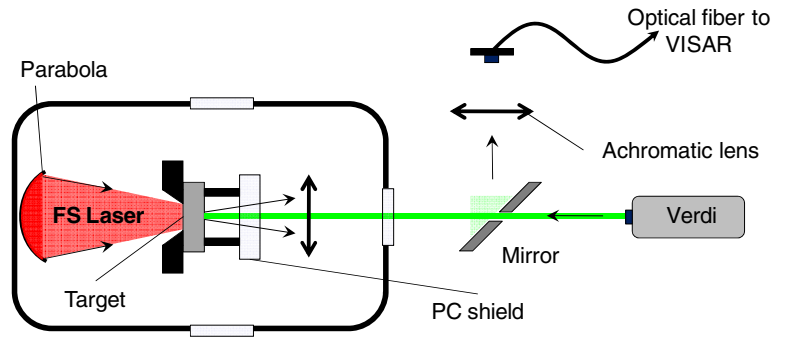

Fig. 1. LULI 100TW Experimental setup.

process is different in fs regime compared to ns regime. In this ultra-short mode, the beam reaches the target and initially excites the electrons on the front face, creating an electron-ion non-equilibrium state. The energy is then transmitted to the crystal lattice by particle collisions along one micron depth. The relaxation time of this imbalance state is about ten picoseconds, which is considerable in comparison of the pulse duration [6]. The imposed gradient of temperature imposed causes phase transitions at high-speed rates, vaporizing and melting the matter which is ejected, leading to an ablation of matter in the micron range [7]. This yields to a pressure profile at the origin of the shock wave. The equivalent pressure loading has been predicted by using the one-dimensional lasermatter interaction code ESTHER provided by the CEA [8]. This code includes particularly a two temperatures model (TTM) describing the electron-ion non-equilibrium state.

Figure 2 represents the average simulated loading for aluminium and tantalum induced by the 100TW laser for intensities included in the utilisation domain. The profile has been picked up at $2 \mu \mathrm{m}$ depth in the target, where this latter has remained solid. It is normalized by its maximum pressure and its middle height duration.

Several simulations at different laser intensities showed the solicitation shape and its middle height duration does not vary for one given material. Thus, it can be characterized by its maximum pressure, given by a power law and its middle height duration, constant for one material. The intensity/maximum pressure values and the profile/laser durations ratios for aluminum and tantalum are reported on the figure 2.

One remarkable feature is that a $300 \mathrm{fs}$ laser pulse generates a pressure profile whose characteristic duration is more than two hundred times greater at FWMH. This dilatation is due to both ion/electron thermal equilibration and the beginning of decay for the wave.

\subsection{Shock wave propagation and attenuation}

In order to check the ability of numerical codes to simulate shock wave propagation in this ultra-short regime, numerical simulations have been performed with the onedimensional hydrodynamic code called SHYLAC. In this short regime, wave propagation is mainly ruled by its attenuation [9]. Numerically, this latter depends on two factors:

- The applied equivalent loading, this comes from lasermatter interaction code.

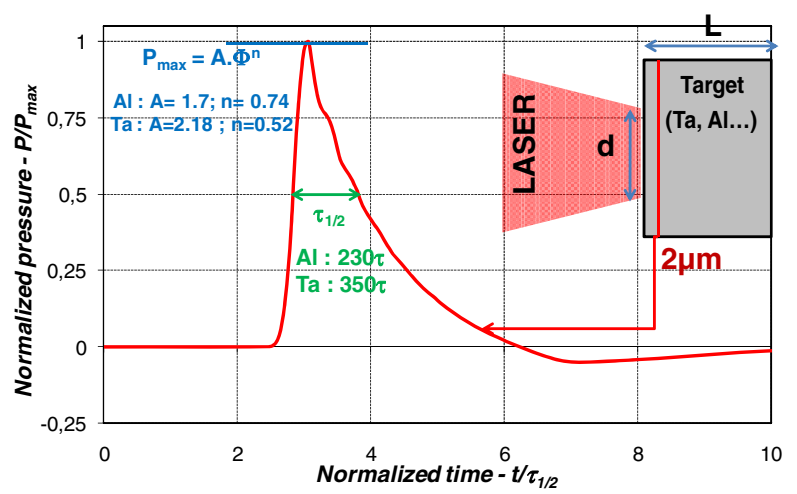

Fig. 2. Normalized pressure loading for the 100TW fs laser calculated with the code ESTHER in the intensity interval $\left[10^{13} ; 10^{15} \mathrm{PW} / \mathrm{cm}^{2}\right]$ (Pressure law units are $\mathrm{PW} / \mathrm{cm}^{2} \&$ Mbar).

- The material law, and particularly the Equation Of State (EOS) and the elastic-plastic behavior.

Shock wave decay can be experimentally followed through the maximum free surface velocity measurement for samples with increasing thickness. This value has been used as attenuation indicator and allows to compare numerical models to experimental points. By this way, it is possible to validate the mechanical behavior simulated with SHYLAC, but also the initial loading from ESTHER generated under the fs laser action. Figure 3 presents some experimental results compared to numerical models in terms of decay.

Figure 3(a) exhibits attenuation curves for aluminum targets with different thicknesses and submitted to similar laser conditions $\left(\tau=300 \mathrm{fs}-\Phi=0.6 \mathrm{PW} / \mathrm{cm}^{2}\right)$. The $\mathrm{Y}$ axis is the ratio between the maximum free surface velocity and two times the material velocity relative to maximum shock pressure in the sample - the maximum loading amplitude in this case. Each decay curve represent a combination of a loading and a material model, and the dots are experimental values red on the VISAR signals like figure 3(c). Here, we can observe the influence of the TTM and the presence of an elastic limit. If the medium is only modeled with a pure hydrodynamic behavior, the decay will be overestimated. In opposition, if the TTM is not used in ESTHER, the initial loading is weaker and the attenuation will be underestimated. Only the association of the TTM in ESTHER and a perfect elastic-plastic behavior in SHYLAC give a good concordance with experimental data.

This combination is conserved in the figure 3(b). In this case, the samples are $100 \mu \mathrm{m}$ thick tantalum targets shocked with different laser intensities. Here, two types of tantalum have been employed: single crystals and polycristallines samples in order to observe the influence of microstructure on the shock response. The average grain size of polycrystalline samples is $50 \mu \mathrm{m}$. We can notice a large difference between the two structures: the maximum free surface velocities are two times weaker for single crystals. This can be attributed to a difference in mechanical properties, particularly the elastic limit which may vary strongly between single- and poly-crystals. The best elastic limits which fits the experimental points are $8 \mathrm{kbar}$ (poly) and 20kbar (single). Concerning the polycrystalline 

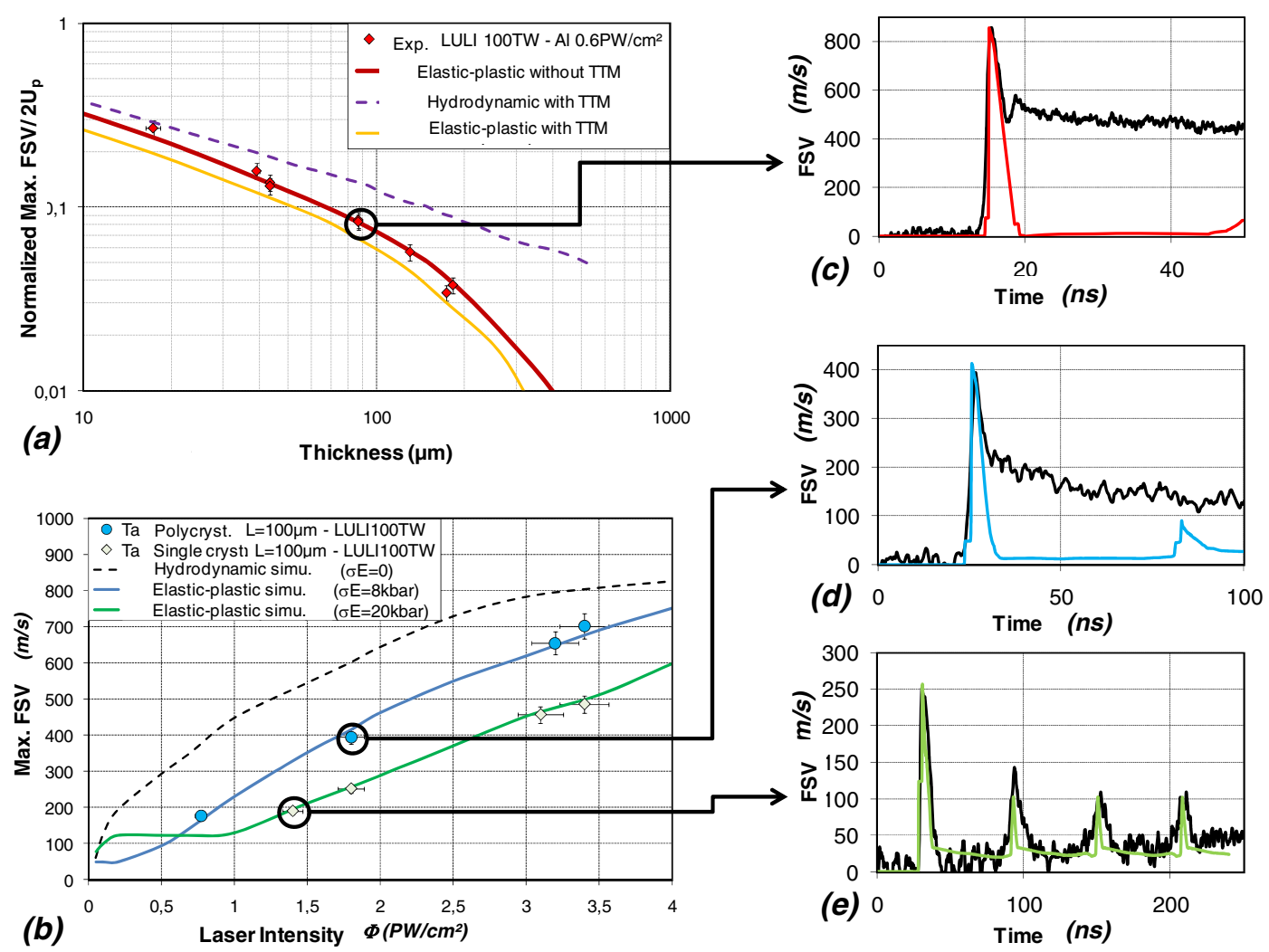

Fig. 3. Num./Exp. Validation of genaration \& attenuation of 100TW fs laser driven shock in aluminum and Tantalum (a) $\Leftrightarrow$ Normalized Maximum Free Surface Velocity (FSV)/Thickness L (Aluminum, 0.6PW $/ \mathrm{cm}^{2}$ ) (b) $\Leftrightarrow$ Maximum Free Surface Velocity/Laser intensity (Ta Single \& polycrystalline $-\mathrm{L}=100 \mu \mathrm{m}$ ) (c) $\Leftrightarrow$ Maximum Free Surface Velocity (Spalled Aluminum L $=100 \mu \mathrm{m}$, LULI $100 \mathrm{TW}$ $\left.0.6 \mathrm{PW} / \mathrm{cm}^{2}\right)(\mathrm{d}) \Leftrightarrow$ Maximum Free Surface Velocity (Spalled polycrystalline Ta $\left.-\mathrm{L}=100 \mu \mathrm{m}, 1.8 \mathrm{PW} / \mathrm{cm}^{2}\right)(\mathrm{e}) \Leftrightarrow$ Maximum Free Surface Velocity (Undamaged single crystal Ta $\mathrm{L}=100 \mu \mathrm{m}, 1.4 \mathrm{PW} / \mathrm{cm}^{2}$ ).

case, the value is in good accordance with the evolution of elastic limit with the strain rate as it can be seen in table 1 .

If the maximum free surface velocity is a good parameter to observe decay, it is necessary to verify the numerical/experimental velocity shapes agreement. Examples of such comparisons are showed on figures 3(c) to (e). They are representative of each material studied $(\mathrm{c}=$ aluminum, $\mathrm{d}=$ polycrystalline $\mathrm{Ta}$ and $\mathrm{e}=$ Ta single crystal), damaged or not. All the numerical curves fit globally the VISAR signals. Only the curve recess which occurs during the release in $\mathrm{c}$ and $\mathrm{d}$ are not reproduced numerically. They are characteristic of rupture by spallation [11] and can only be simulation by adding a damage model. This is going to be discussed in the next paragraph.

\section{Dynamic damage modeling}

\subsection{Kanel dynamic damage model}

We have integrated into the previously validated numerical model a damage criterion in order to represent the spallation induced by the shock wave reflection. We chose the model of Kanel, based on the growth of a void rate in an elementary volume starting from a given threshold in tension and described by the following differential
Table 1. Evolution of tantalum elastic limit with strain rate [10] (the bold value correspond to this work).

\begin{tabular}{|c|c|}
\hline Strain rate $\left(\mathrm{s}^{-1}\right)$ & Elastic limit $(\mathrm{MPa})$ \\
\hline $10^{-4}$ (Quasi-static) & 180 \\
\hline $10^{2}$ (Hopkinson bar) & 310 \\
\hline $2.10^{4}$ (Plate impact) & 550 \\
\hline $\mathbf{8 , 2 . 1 0}^{\mathbf{6}}$ (fs laser) & $\mathbf{8 0 0}$ \\
\hline
\end{tabular}

equation (1) [12].

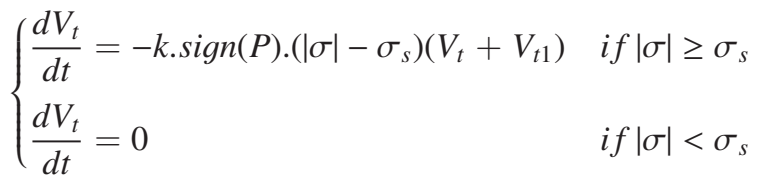

Where $V_{t}$ is the void rate, $\sigma$ the axial stress, $\sigma_{s}$ the threshold stress and $P$ the pressure. $k$ and $V_{t 1}$ are characteristic of the material. The evolution of the void rate retroacts on the material mechanical properties (equations (2) to (4)):

$$
\begin{gathered}
\sigma_{s}=\sigma_{s}^{0} \cdot \frac{V_{t 1}}{V_{t 1}+V_{t}} \\
G=G^{0} \cdot \frac{V_{t 1}}{V_{t 1}+V_{t}}
\end{gathered}
$$



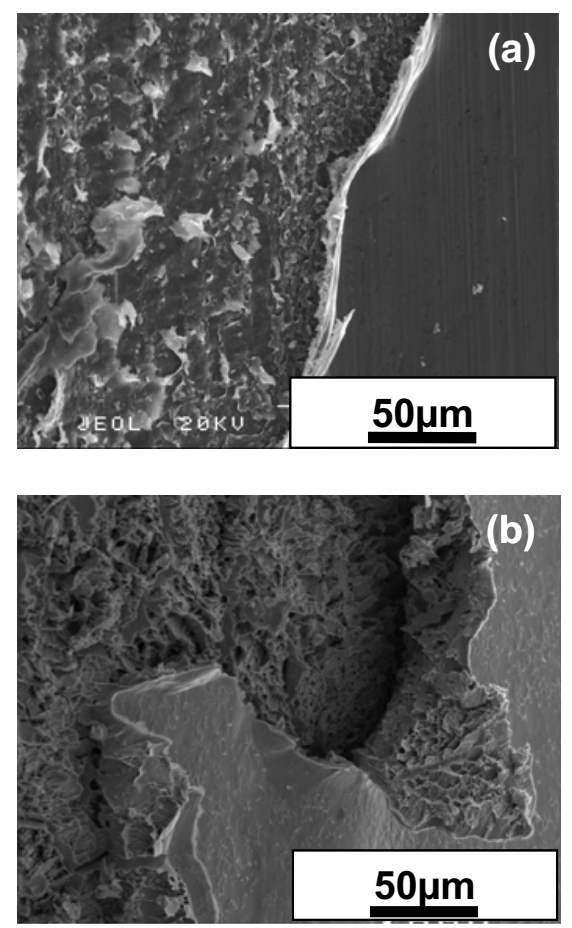

Fig. 4. Post-shock SEM pictures of the rear surface of two spalled target including the rupture plan.

(a) $\Leftrightarrow 100 \mu \mathrm{m}$ thick Aluminum $-\Phi=0.6 \mathrm{PW} / \mathrm{cm}^{2}$

(b) $\Leftrightarrow 100 \mu \mathrm{mm}$ thick polycrystalline tantalum $-\Phi=$ $3.4 \mathrm{PW} / \mathrm{cm}^{2}$.

$$
Y=Y^{0} \cdot \frac{V_{t 1}}{V_{t 1}+V_{t}}
$$

Where $\sigma_{d}^{o}, \mathrm{G}^{o}, \mathrm{Y}^{o}$ are respectively the initial threshold stress, shear modulus and yield strength. One of the main advantages of this model is the number of parameter to find, only three. This model is thus adapted to describe ductile rupture, which is the fracture mode in this work. This is confirmed by looking at the sample after shock in the rupture plan created by spallation. Examples of ductile spalled plan are presented on Figure 4 which are SEM pictures of both aluminum and tantalum rear surfaces (respectively 4(a) and 4(b)). The aim in this study is thus to estimate the Kanel parameters at the strain rates associated to the fs regime. They can be determined by fitting the last part of experimental VISAR signals (the final velocity plateau corresponding to the spall).

\subsection{Kanel parameters calibration with strain rate}

Figures 4 and 5 show two examples of Kanel fitting for respectively a $150 \mu \mathrm{m}$ thick aluminum target submitted to a $0.6 \mathrm{PW} / \mathrm{cm}^{2}$ and a $120 \mu \mathrm{m}$ single crystal tantalum target at $3.4 \mathrm{PW} / \mathrm{cm}^{2}$. For the aluminum case, we have tested two rupture criteria: Kanel and a simple Cut-off (instantaneous rupture when spall strength $\sigma_{R}$ is reached, here $\sigma_{R}=15 \mathrm{kbar}$ ).

It can be noticed that a simple stress threshold is unable to reproduce the signal evolution after the accident

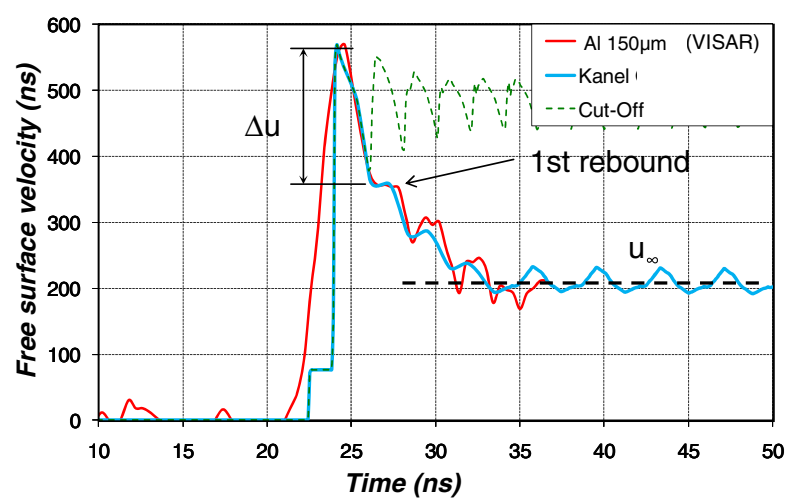

Fig. 5. Comparison of experimental and numerical free surface velocities with the Cut-off and Kanel damage models $\left(150 \mu \mathrm{m}\right.$ thick aluminum $\left.-\Phi=0.6 \mathrm{PW} / \mathrm{cm}^{2}\right)$.

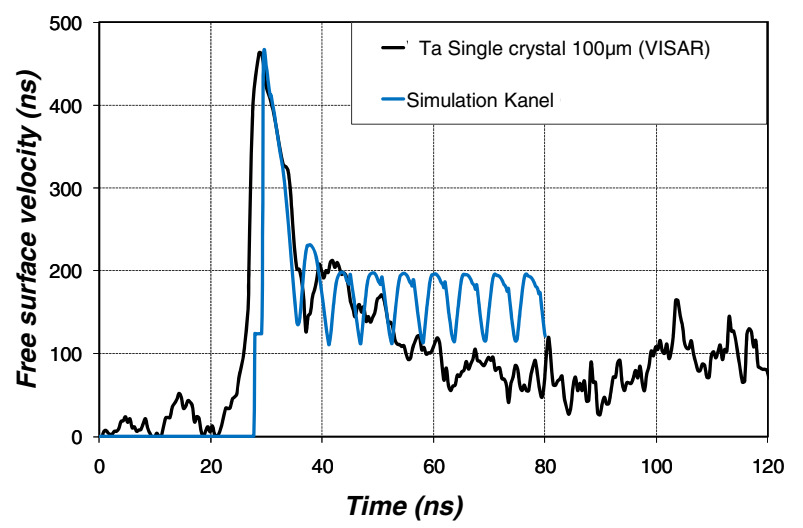

Fig. 6. Comparison of experimental and numerical free surface velocities with the Kanel model $(100 \mu \mathrm{m}$ thick single crystal tantalum $-\Phi=3.4 \mathrm{PW} / \mathrm{cm}^{2}$ ).

characteristic of rupture: the average spall velocity is overestimated in this case. Therefore, Kanel model reproduce well the curve when an adequate parameter set is found (here we obtain: $\sigma_{\mathrm{s}}=15.5 \mathrm{kbar} ; \mathrm{k}=2.3 \mathrm{~Pa}^{-1} . \mathrm{s}^{-1} ; V_{\mathrm{t} 1}=$ 0.08).

Kanel parameter sets have been calibrated for aluminum and polycrystalline tantalum samples with different thicknesses. The parameters dimensioning implies a good restitution of three spall signal characteristic features (see figure 3):

- The velocity pullback amplitude $\Delta \mathrm{u}$

- The amplitude of the first following rebound

- The final average spall velocity $\mathrm{u}_{\infty}$

However, we obtain for a same material different parameter values. Moreover, they are all higher than sets found in the literature for nanosecond laser driven shock (In [3] : $\left.\sigma_{s}=15 \mathrm{kbar} ; k=0.23 \mathrm{~Pa}^{-1} \mathrm{~s}^{-1} ; V_{\mathrm{t} 1}=0.03\right)$.

This shows that the Kanel parameters vary with the solicitation nature, in particular strain rate $\left(\sim 10^{6} \mathrm{~s}^{-1}\right.$ in ns regime vs. $10^{7} \mathrm{~s}^{-1}$ for fs regime). In the case of femtosecond regime, the strain rate may change strongly with the conditions. Indeed, an increase in target thickness will change the tension time history along the rupture plane which will be slower because of wave attenuation (similarly to what happens during the loading generation just after laser-matter interaction). 

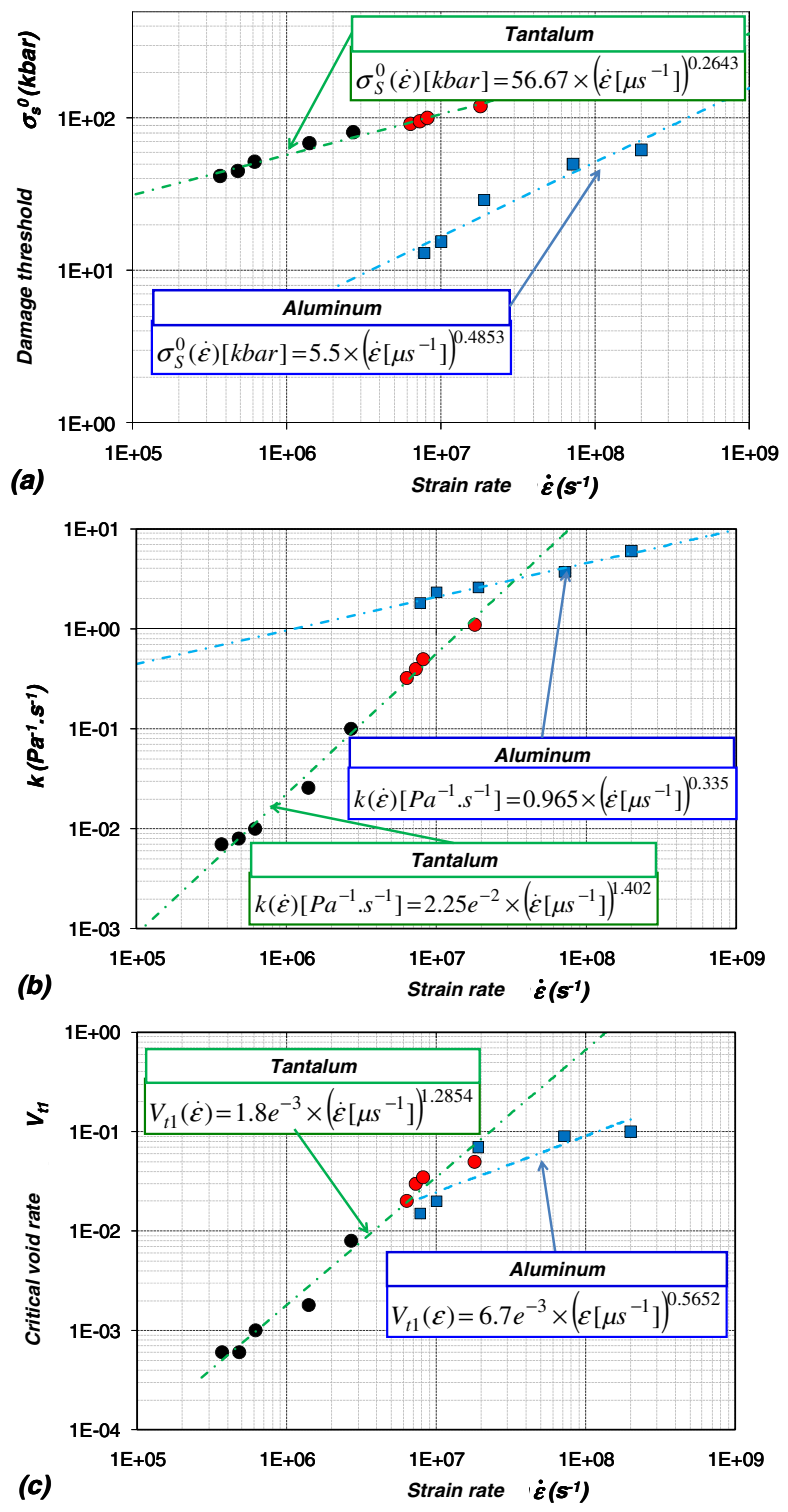

Fig. 7. Normalize the.

Strain rate can be estimated from VISAR signals by using equation (5):

$$
\dot{\varepsilon}=\frac{\Delta u}{\Delta t} \cdot \frac{1}{2 \cdot c_{o}}
$$

Where $\Delta \mathrm{u}$ is the velocity pullback amplitude and $\Delta \mathrm{t}$ its duration. $\mathrm{C}_{0}$ stands for the speed of sound in the considered material. If the target thickness is enlarged, $\Delta \mathrm{t}$ (similar to the tension loading duration) and $\Delta \mathrm{u}$ (proportional to $\sigma_{\mathrm{s}}$ ) will increase and strain rate too [2]. This fact opens the possibility to study damage mechanics at very high strain rates $\left(10^{7}\right.$ to $10^{8} \mathrm{~s}^{-1}$ in fs regime). The strain rate comparison can be achieved by using transversally several shock generators. Figure 7 shows the evolution of Kanel parameter obtained for various sample thickness - so different strain rates - in both materials. When strain rate is increased, all the parameters increase. Black dots plotted for tantalum correspond to other shock wave generators including plate impacts and ns laser driven shocks.
For each material, all the parameters can be interpolated by a power law type with a good agreement overall.

Hence, it is possible to expand the classical Kanel law to a generalized dynamical model which takes into account strain rate through a power law type for its parameters.

\section{Conclusions}

Laser driven shock experiments on tantalum and aluminum targets performed with a subpicosecond source showed the possibility to investigate the material behavior under extreme strain rates in the whole. The acquisition of the free surface velocity allowed to validate the generation of an intense and short pressure wave consequently to the laser-matter interaction. It showed that some ultra-brief phenomena have to taken into account (as electron-ion imbalance), contrary to longer regime where compression is ruled mainly by the plasma expansion. Also, such experiments highlighted the importance of material modeling to reproduce correctly the shock wave propagation. It appeared the elastic-plastic behavior plays a non-negligible role in the wave attenuation. The comparison between experimental and numerical free surface velocities showed that an ultra-short irradiation is in the continuity of shocks physics into this extremely short duration scales, from shock propagation to spallation.

We shown that Kanel damage model was able to reproduce well the ductile rupture of tantalum. The strain rate model dependence has been evidenced with a power law for all the parameters. By fitting these latter with experimental results from several shock generators which have different strain rates domains, we obtained a generalization of the Kanel model for a large dynamic strain rates field.

Based on these encouraging results, it is planned to complete the elaboration of a generalized model. In a first time, we would enlarge the validity interval with slower strain rates corresponding to Hopkinson bar or even quasistatic experiments. On the other side, Molecular Dynamic simulations provide an original way to predict the behavior at ultra-high strain rates and would open be associated with microscopic study of damage by spallation in order to understand micro-mechanisms responsible of ductile damage at these extreme solicitations [13]. Finally, the model could be more generalized by taking into account temperature effects whose effects could be equivalent to an inverse variation in strain rate.

\section{Acknowledgements}

We are very grateful to all the LULI staff who helped in building these experiments and trained us to run the 100TW laser facility : P. Audebert, J. Albrecht, J. Fuchs, C. Le Bris, Ji Ping Zou, S. Baton.... Many thanks to the CEA researchers from DPTA/CEA-DIF for joining this work through the expertise of D Van der Hagen, and particularly for the numerical part with "CEA/ESTHER" code kindly provided by CEA to our labs. 


\section{References}

1. R. Kinslow, High velocity impact phenomena Academic Press, New-York/London, (1971).

2. E. Moshe et al, J. Appl. Phys., 83, 4004, (1998).

3. L. Tollier, R. Fabbro and E. Bartnicki, J. Appl. Phys., 83(3), 1231, (1998).

4. C. Rulliere, Femtosecond Laser Pulses - Principles and experiments, Springer, New-York, (2003).

5. H. Tamura, T. Kohama, K. Kondo et al, J. Appl. Phys., 89, 3520, (2001).

6. J.-P. Cuq-Lelandais, M. Boustie, L. Berthe et al, J. Phys D, 42, 065402, (2009).

7. Elsayed-Ali H.E., Norris T.B., Pessot M.A. and Mourou G.A., Phys. Rev. Lett. 68, 2834 (1992).
8. J.-P. Colombier, P. Combis, R. Stoian and E. Audouard, Phys. Rev. B, 75, 104105 (2007).

9. Bonneau F. et al, Appl Phys B :Lasers Opt., B78, 447, (2004).

10. D.J. steinberg and C.M. Lund, J. Phys. Colloques, 49 C3-433, (1988).

11. T. Antoun, L. Seaman, D.R. Curran et al, Spall fracture, Springer-Verlag, New-York, (2002).

12. G.I. Kanel and V.E. Fortov, Usp. Mekh., 10(3) 3, (1987).

13. L. Soulard, J. Bontaz-Carion, J.-P. Cuq-Lelandais, M. Boustie, T. De Rességuier and A. Sollier, New Models and Hydrocodes for Shock wave processes in condensed matter, Paris - France (2010). 